\title{
The Combination of Dysexecutive and Amnestic Deficits Strongly Predicts Conversion to Dementia in Young Mild Cognitive Impairment Patients: A Report from the Gothenburg-Oslo MCI Study
}

\author{
Erik Hessen $^{a, b}$ Ivar Reinvang ${ }^{b} \quad$ Carl F. Eliassen ${ }^{a, b} \quad$ Arto Nordlund $^{e}$ \\ Leif Gjerstad ${ }^{c}$ d Tormod Fladby ${ }^{a, c}$ Anders Wallin ${ }^{e}$ \\ ${ }^{a}$ Department of Neurology, Akershus University Hospital, ${ }^{b}$ Clinical Neuroscience \\ Research Group, Department of Psychology and ${ }^{\mathrm{C} F a c u l t y}$ of Medicine, University of \\ Oslo, and ${ }^{\mathrm{d}}$ Department of Neurology, Oslo University Hospital, Oslo, Norway; \\ e Institute of Neuroscience and Physiology, Sahlgrenska Academy at Gothenburg \\ University, Mölndal, Sweden
}

\section{Key Words}

Dysexecutive mild cognitive impairment - Amnestic mild cognitive impairment .

Multidomain mild cognitive impairment - Prognosis of mild cognitive impairment

\begin{abstract}
Background/Aims: The present study aimed to add to the knowledge of mild cognitive impairment $(\mathrm{MCI})$ by studying the prognosis in a relatively young cohort of patients characterized by neuropsychological criteria. Methods: Patients (mean age: 63 years) with cognitive complaints and MCI $(n=302)$ were recruited from two university clinics and followed for 2 years. Results: Pure dysexecutive $\mathrm{MCI}$ occurred in $11.7 \%$ of the neuropsychologically impaired patients, while 59.3 and $29.0 \%$ were characterized as having pure amnestic MCI or multidomain MCI. During the study period, the state of $2(10.5 \%)$ of the patients with single-domain dysexecutive MCI converted to dementia, while 28 (29.2\%) of the patients with pure amnestic MCI became demented. Of the patients with both executive and amnestic deficits, 28 (59.6\%) became demented. Conclusion: The results suggest that dysexecutive symptoms in combination with amnestic symptoms constitute a strong risk factor for dementia in young MCI patients. A significant number of patients in all subgroups showed normal test results at followup, indicating that a neuropsychological diagnosis needs to be supported by imaging or biomarker data.




\section{Introduction}

It is well recognized that Alzheimer's disease (AD) and cerebrovascular disease processes may start years before cognitive symptoms become manifest [1,2] and that there is a pressing need to find biological and cognitive markers both in order to predict future clinical decline and for use as outcome measures in clinical trials of possible disease-modifying agents.

In 1999 researchers from the Mayo Clinic proposed quantitative criteria for identifying mild cognitive impairment (MCI) as a prodromal stage of $\mathrm{AD}$ [3], focusing on the importance of memory impairment. Amnestic MCI is characterized by a clinically significant memory deficit, and the probability of progression from amnestic MCI to dementia is approximately 5-10\% per year $[4,5]$. The Alzheimer's Disease Neuroimaging Initiative (ADNI) project [1] has yielded a wealth of clinical information on pathophysiology and prognosis in patients with amnestic MCI at a mean age between 70 and 80 years. Several studies have shown that the rate of progression is likely to be more rapid with greater neuropsychological impairment at baseline [6, 7].

Nonamnestic forms of $\mathrm{MCI}$ are characterized by subtle but clinically significant deficits in attention, executive functions, language and visuospatial abilities [8]. The nonamnestic forms are probably less common than the amnestic type, which is consistent with findings in the Mayo Clinic Study of Aging, where two thirds of the persons with MCI (70-89 years) were characterized as amnestic MCI cases [8]. Nonamnestic MCI is diverse in cognitive deficits and in its underlying pathophysiology [9], and recent studies that include neuropsychological testing [10-17] have focused on dysexecutive MCI as a clinically significant subtype.

The test batteries, definition of executive functions and inclusion criteria vary in all these studies, and so does the prevalence of nonamnestic dysexecutive $\mathrm{MCI}$, ranging from 3 to $15 \%$ [18]. Although the occurrence of nonamnestic dysexecutive MCI may be limited compared with amnestic $\mathrm{MCI}$, a possible decline in executive functions is of considerable importance as it is related to poorer function in activities of daily living [19], and because it has been shown to predict conversion from MCI to dementia $[6,17]$.

MCI is also a preliminary stage of progressive cerebrovascular disease, but memory loss is usually not the predominant manifestation of this disease process. Instead, speed/attention and dysexecutive impairments have been found to be characteristic features of vascular cognitive impairment $[2,20,21]$. However, various forms of $\mathrm{MCI}$ in this group of patients have not yet been described.

Dysexecutive nonamnestic MCI may be associated with a variety of other conditions including early stages of frontotemporal dementia [22], Parkinson's disease [23], dementia with Lewy bodies [24], prodromal stages of $\mathrm{AD}[17,25-27]$ and neuropsychiatric conditions (depression) [28]. As the frequency of dysexecutive symptoms is high in patients with manifest small vessel disease [29], mild dysexecutive impairment may be showing in persons with subclinical vascular pathology commonly found in the older population [30]. These are different conditions with different prognoses and their pathophysiological bases both vary and remain partly unclear [31,32].

The Gothenburg-Oslo MCI Study (GO-MCI) includes mainly patients in their mid-60s with MCI broadly defined as subjective or objective cognitive impairment. In a 2-year follow-up of 209 MCI patients, Nordlund et al. [33] found that of the 44 patients (25\%) who developed dementia, 35 originated from a multidomain amnestic group and 9 belonged to a multidomain nonamnestic group. The results from the study suggest that impairment in multiple cognitive domains is associated with a more severe outcome over 2 years. Furthermore, the results suggest that single-domain impairment (memory or other cognitive functions) is a rather benign condition in this young MCI cohort. Finally, 20\% of those who progressed to dementia did not show memory impairment at baseline, suggesting that memory impairment is not always the first symptom of dementia disorders. 
Hessen et al.: The Combination of Dysexecutive and Amnestic Deficits Strongly

Diverse findings in previous research imply that the potential predictive role of nonamnestic dysexecutive $\mathrm{MCI}$ is far from settled. There is no consensus regarding tests and criteria defining the group. The cited studies employ different individual measures of executive functions, combine executive and nonexecutive measures, employ different composite measures and different cutoff criteria for impairment and study different age groups, and many include only a small number of participants. Furthermore, the concept of executive function is heterogeneous, containing several subdomains [34], some of which may have greater predictive significance than others [35, 36]. Brandt et al. [35] found that working memory and problem solving, but not judgment, were impaired in MCI patients, including amnestic MCI. Johnson et al. [13] found deficits in several aspects of executive function in MCI patients, but inhibitory control was the most frequently and severely impaired.

The present study aimed to add to the knowledge of MCI by studying its prognosis in a relatively young cohort of patients characterized by neuropsychological criteria, with singledomain amnestic MCI, single-domain dysexecutive MCI and multidomain deficit MCI (amnestic $\mathrm{MCI}+$ dysexecutive MCI). Furthermore, the prognosis of MCI was compared with clinical controls (memory clinic patients with normal neuropsychological status). We hypothesized that the combination of executive dysfunction and amnestic problems at baseline would increase the risk of conversion to dementia.

\section{Materials and Methods}

The Norwegian part of the project was approved by the South-Eastern Norwegian committee for medical research ethics. The Swedish part of the study was approved by the local ethics committee, and the research at both sites was conducted according to the Helsinki Declaration.

Participants were recruited from the GO-MCI, which is a collaborative longitudinal study of people with MCI. The memory clinics at Sahlgrenska University Hospital in Gothenburg and Akershus University Hospital in Oslo employ the same inclusion and exclusion criteria, similar clinical assessments and similar neuropsychological test batteries.

About three quarters of the participants were referred by their general practitioners or a medical specialist, while about a quarter were self-referrals. People with major depressive and other severe psychiatric disorders were excluded, while participants with minor depressive symptoms and mild anxiety were not. Additionally, persons with neurological disease, a history of traumatic brain injury, cardiovascular accident, other severe somatic illness or current substance abuse were excluded. The diagnosis of MCI was made in congruence with the most recent recommendations and has been described elsewhere [14, 37]. To summarize, MCI was diagnosed by means of a medical history and checklists for cognitive symptoms: Stepwise Comparative Status Analysis [14] (including memory disturbance, disorientation, reduced abstract thinking, visuospatial disturbance, poverty of language, sensory aphasia, visual agnosia and apraxia); I-Flex [14], which is an interview for executive symptoms (including screening test items number-letter task, word fluency, anomalous sentence repetition, interference task, Luria hand sequences and counting task); MiniMental State Examination (MMSE), and Clinical Dementia Rating (CDR). The information for CDR was gathered from both the subject and an informant. For inclusion, subjective and objective evidence (verified by an informant) was required for progressive cognitive impairment over a period of more than 6 months. Furthermore, objective cognitive symptoms according to the procedure described above were required. Subjects without symptoms according to the checklists or CDR were not included, since their cognitive impairment was considered too benign, and neither were subjects with more than 2 cognitive symptoms on 
the described checklists and/or a score below 25 on the MMSE, as they were considered to fulfill criteria for dementia. The included patients were classified by the Global Deterioration Scale (GDS) [38, 39] with scores of 2 and 3, as determined by the procedure described above. From the GO-MCI database, we identified MCI patients with amnestic, nonamnestic executive and multidomain impairment at baseline evaluation, based on neuropsychological criteria (see below).

In the present analysis we included the 4 tests of executive function and memory that were most frequently used at both sites. This included 2 memory tests, the Rey Auditory Verbal Learning Test (RAVLT) $[40,41]$ and visual reproduction from the Rey Complex Figure Test (RCFT) [42], and 2 executive measures, the Trail Making Test B (TMT-B) [43] and verbal fluency (Controlled Oral Word Association Test, COWAT) [44]. All the test results were converted to T scores, a normally distributed scale with a mean score of 50 and a standard deviation (SD) of 10. We created an 'executive index', based on the TMT-B and COWAT, and an 'amnestic index', consisting of RAVLT-delayed recall and RCFT-delayed reproduction. Additionally, we created a 'total impairment index', consisting of all 4 tests included. A T score of 37 or below was given an index score of 1, resulting in 2 indexes (executive and amnestic) ranging from 0 to 2 and 1 index (total impairment) ranging from 0 to 4 . Dysexecutive, amnestic and multidomain MCI were defined solely by these neuropsychological test scores. Patients scoring 1 or 2 on either the executive or amnestic index and with no scores below the cutoff $(\mathrm{T}=37)$ on the other index were characterized as cases of dysexecutive MCI or amnestic MCI. Patients scoring below the cutoff criterion on 1 or 2 tests from both indexes were defined as cases of multidomain MCI. A cutoff criterion of $\mathrm{T}=37$ has been employed in several previous studies of nonamnestic dysexecutive MCI $[10,11,15,16]$. The rate of conversion to dementia after 2 years (GDS score 4 or higher) was analyzed for each of the MCI subtypes.

\section{Normative Data}

The RAVLT was scored by the norms provided by Schmidt [41]. Reproduction of the RCFT was scored by the normative data from Meyers and Meyers [42]. The TMT-B and the COWAT were scored by the demographic norms by Heaton et al. [45]. Conversion of T scores was based on these normative data.

\section{Statistical Analysis}

The Statistical Package for the Social Sciences (SPSS version 19.0) was used for all statistical analyses. First, descriptive statistics for the demographic, clinical and cognitive characteristics of the patient population were computed. Then, a series of independent $t$ tests was performed, comparing the single-domain MCI groups as well as the multidomain MCI group with the group showing normal test results (noMCI group). Odds ratios (OR) for development of dementia were estimated by logistic regression analysis. First, the neuropsychological MCI categories described above were tested in a logistic univariate model. Each of the MCI groups was compared with all the other groups combined. Based on this analysis, independent variables were chosen to be tested in a multivariate logistic model. Crosstab statistics was then performed to describe the development of the different MCI categories over a 2-year period.

\section{Results}

\section{Demographic and Clinical Characteristics}

The demographic and clinical variables of the 302 patients who took part in the study are shown in table 1 . The baseline test scores for patients with no MCI compared with the MCI subtypes, based on neuropsychological criteria, are given in table 2. For the group charac- 
Table 1. Baseline characteristics of all study patients $(n=302)$

\begin{tabular}{l|l}
\hline \multicolumn{2}{l}{ Dement Geriatr Cogn Disord Extra 2014;4:76-85 } \\
\hline DOI: $10.1159 / 000360282$ & $\begin{array}{l}\text { ○ 2014 S. Karger AG, Basel } \\
\text { www.karger.com/dee }\end{array}$ \\
\hline $\begin{array}{l}\text { Hessen et al.: The Combination of Dysexecutive and Amnestic Deficits Strongly } \\
\text { Predicts Conversion to Dementia in Young Mild Cognitive Impairment Patients }\end{array}$ \\
\hline \\
\hline Age, years & $62.7 \pm 7.5$ \\
Female, n (\%) & $166(54.9)$ \\
Education, years & $12.2 \pm 3.4$ \\
GDS score & $2.7 \pm 0.5$ \\
MMSE score & $28.4 \pm 1.3$ \\
RAVLT - delayed recall, T score & $44.2 \pm 13.4$ \\
RCFT - delayed reproduction, T score & $43.8 \pm 15.4$ \\
COWAT, T score & $49.7 \pm 10.9$ \\
TMT-B, T score & $47.3 \pm 10.1$ \\
Executive index & $0.26 \pm 0.51$ \\
Amnestic index & $0.67 \pm 0.78$ \\
Total impairment index & $0.91 \pm 1.0$ \\
\hline
\end{tabular}

Values denote means \pm SD unless specified otherwise.

Table 2. Baseline performance of patients with no MCI compared with MCI subtypes based on neuropsychological criteria

\begin{tabular}{lllll}
\hline & noMCI $(\mathrm{n}=140)$ & eMCI $(\mathrm{n}=19)$ & aMCI $(\mathrm{n}=96)$ & mMCI $(\mathrm{n}=47)$ \\
\hline MMSE, raw score & $28.7 \pm 1.2(5)$ & $28.2 \pm 1.2(4)^{*}$ & $28.1 \pm 1.4(5)^{* *}$ & $28.1 \pm 1.4(5)^{* *}$ \\
RAVLT - delayed recall, T score & $52.8 \pm 9.2(46)$ & $47.5 \pm 7.8(29)^{*}$ & $36.2 \pm 11.7(47)^{* *}$ & $33.8 \pm 11.5(56)^{* *}$ \\
RCFT - delayed reproduction, T score & $54.3 \pm 10.6(56)$ & $51.9 \pm 13.1(58)$ n.s. & $33.5 \pm 10.9(52)^{* *}$ & $30.3 \pm 11.4(36)^{* *}$ \\
COWAT, T score & $53.7 \pm 9.1(37)$ & $39.9 \pm 8.1(28)^{* *}$ & $51.0 \pm 9.3(43)^{*}$ & $39.8 \pm 11.1(37)^{* *}$ \\
TMT-B, T score & $51.0 \pm 7.8(45)$ & $35.1 \pm 11.9(50)^{* *}$ & $49.4 \pm 7.2(36)$ n.s. & $37.1 \pm 9.3(48)^{* *}$ \\
Impairment index & $0.0 \pm 0.0$ & $1.2 \pm 0.4^{* *}$ & $1.4 \pm 0.5^{* *}$ & $2.5 \pm 0.8^{* *}$ \\
\hline
\end{tabular}

Values denote means \pm SD with maximum ranges in parentheses. $* p<0.05,{ }^{* *} \mathrm{p}<0.01$. eMCI $=$ Dysexecutive MCI; aMCI $=$ amnestic MCI; mMCI = multidomain MCI; n.s. = not significant.

terized as noMCI, all the T scores were close to or slightly above the normative mean. The same group also achieved a normal score on the MMSE and the normal impairment index. The MCI subtypes were compared with the noMCI group, with significant differences evident particularly in the domains specific for each MCI subtype. Scores on the MMSE, on the impairment index as well as on the individual tests gradually decreased, with dysexecutive MCI scoring better than amnestic MCI and with multidomain MCI scoring clearly poorer than the other MCI groups.

\section{Prediction of Dementia}

Each of the MCI groups was compared with all the other groups combined. Univariate logistic regression analysis revealed a significant association between multidomain MCI and amnestic MCI, and development of dementia (GDS score $\geq 4$ ) after 2 years (table 3). Multivariate regression analysis showed that both multidomain $\mathrm{MCI}(\mathrm{OR}=22.25, \mathrm{p}=0.001)$ and amnestic MCI (OR $=5.91, \mathrm{p}=0.001)$ were significant and strong predictors of dementia at follow-up.

The classification at baseline and development of MCI subtypes over 2 years are shown in table 4. At baseline, 19 (11.7\%) of the neuropsychologically impaired patients showed pure dysexecutive MCI, 96 (59.3\%) showed pure amnestic MCI and a further 48 (29.0\%) of the total MCI patients showed executive deficits combined with amnestic impairment. As expected, patients scoring poorly at baseline showed a greater tendency toward conversion to dementia, defined as a GDS score of 4 or above: 28 (29.2\%) showed conversion from 
Table 3. MCI subtypes as predictors of dementia (GDS $\geq 4$; $\mathrm{n}=302$ )

\begin{tabular}{|c|c|c|c|c|c|}
\hline & \multicolumn{2}{|c|}{ Univariate analysis } & \multicolumn{3}{|c|}{ Multivariate analysis } \\
\hline & OR & $\mathrm{p}$ & OR & $95 \%$ CI & $\mathrm{p}$ \\
\hline Multidomain MCI & 8.76 & 0.001 & 22.25 & $9.37-52.87$ & 0.001 \\
\hline Amnestic MCI & 1.75 & 0.053 & 5.91 & $2.71-12.88$ & 0.001 \\
\hline Executive MCI & 0.42 & 0.247 & & & \\
\hline No MCI & 0.11 & 0.001 & & & \\
\hline
\end{tabular}

Table 4. Development of MCI subtypes over 2 years

\begin{tabular}{|c|c|c|c|c|c|c|}
\hline & \multirow[t]{2}{*}{ Baseline } & \multicolumn{5}{|c|}{ Follow-up after 2 years } \\
\hline & & noMCI & eMCI & aMCI & $\mathrm{mMCI}$ & dementia/GDS 4 \\
\hline noMCI & $140(46.4)$ & $110(78.6)$ & $6(4.2)$ & $15(10.7)$ & $3(2.1)$ & $6(4.3)$ \\
\hline eMCI & $19(6.3)$ & $7(36.8)$ & $7(36.8)$ & $1(5.3)$ & $2(10.5)$ & $2(10.5)$ \\
\hline aMCI & $96(31.8)$ & $32(33.3)$ & $1(1.0)$ & $30(31.6)$ & $5(5.2)$ & $28(29.2)$ \\
\hline mMCI & 47 (15.6) & $2(4.3)$ & $4(8.5)$ & $6(12.8)$ & 7 (14.9) & $28(59.6)$ \\
\hline Total & $302(100)$ & $151(50.0)$ & $18(5.9)$ & $52(17.2)$ & $17(5.6)$ & $64(21.2)$ \\
\hline
\end{tabular}

Values denote numbers with percentages in parentheses. $\mathrm{eMCI}=$ Dysexecutive $\mathrm{MCI}$; $\mathrm{aMCI}=$ amnestic $\mathrm{MCI}$; mMCI = multidomain MCI.

amnestic MCI to dementia, while 28 (59.6\%) showed conversion from multidomain MCI to dementia. Several of the MCI patients also improved their performance, and $151(50.0 \%)$ were characterized as normal after 2 years, compared with $140(46.4 \%)$ at baseline. Among those who improved, 7 (36.8\%) of the dysexecutive MCI group and 32 (33.3\%) of the amnestic $\mathrm{MCI}$ group were classified as noMCI at follow-up. It is also of interest that $12(25.5 \%)$ of the patients with multidomain MCI at baseline improved their cognitive function and were characterized as either noMCI, dysexecutive MCI or amnestic MCI after 2 years.

\section{Discussion}

The present study found that executive or amnestic deficits, either in pure form or in combination, occur frequently in a relatively young clinical population referred for cognitive complaints. Only 2 of the patients with single-domain dysexecutive $\mathrm{MCI}$ converted to dementia during the study period. However, dysexecutive symptoms increased the risk of dementia in a multidomain context, suggesting that dysexecutive symptoms in combination with amnestic symptoms are a clear risk factor for dementia in young MCI patients. Pure amnestic impairment was also significantly associated with development of dementia, but to a lesser degree than multidomain impairment. Additionally, while several of the patients progressed from mild to more severe forms of MCI, it is important to note that within the pure dysexecutive and amnestic categories, 36.8 and $33.3 \%$, respectively, improved cognitively from baseline to follow-up.

The findings are based on a selected group of patients recruited at two university memory clinics and diagnosed with subjective cognitive impairment or MCI based on GDS scores of 2 and $3[3,38,39]$. The mean age of the participants was 62.7 years at baseline testing, which 
Hessen et al.: The Combination of Dysexecutive and Amnestic Deficits Strongly

is much younger than in other key clinical studies of $\mathrm{MCI}[8,46]$. In this age group the expected prevalence of dementia is very low, i.e. approximately 160 per 100,000 or less than $0.2 \%$ [47]. As a decline in attention and executive function is common in normal aging [48], some of the study patients with no or only mild executive impairment may simply be low-functioning healthy persons or persons with either subclinical vascular pathology commonly found in an older population [30] or small vessel disease [29].

The present group included patients who progressed to dementia and possibly also patients at early, subclinical stages of dementia that may develop at older age. However, as $50 \%$ of the participants did not show evidence of cognitive impairment - and many also improved - at follow-up, there is indication that the sample consisted of a large number of cognitively healthy persons at low risk of developing dementia in the short time period of the present study. Practice effects might also explain some of the improvement in test scores, even though the executive and recall tests employed usually do not show significant improvement in normal control subjects between 1 and 2 years after baseline assessment [49]. One may also question the reliability and robustness of the dysexecutive and the amnestic groups as they were categorized based on only two tests each. Standard neuropsychological assessment requires more tests in each domain [50] in order to avoid faulty categorization based on single and possibly incidental poor scores.

Both the young age and the relatively mild degree of cognitive impairment in this group imply that the findings are difficult to compare directly with other studies of patients with MCI. Nevertheless, many of the present findings confirm those of other studies of older MCI populations. Employing neuropsychological criteria, both Bozoki et al. [51] and Tabert et al. [6] found that deficits in both memory and psychomotor speed/executive function were a stronger predictor of conversion to $\mathrm{AD}$ than pure amnestic MCI after approximately 2 and 4 years, respectively. In our sample, $59.6 \%$ of those defined as having multidomain MCI converted to dementia, as opposed to $29.2 \%$ of those with amnestic MCI.

Despite similar MMSE scores at baseline in the amnestic MCI and multidomain MCI groups, the latter group - with poorer neuropsychological test scores - clearly indicated more severe cognitive impairment than the single-domain amnestic MCI group. This suggests that the progression toward dementia was more advanced in the multidomain MCI group already at baseline.

Our findings regarding the amnestic group may be questioned. The purity of amnestic $\mathrm{MCI}$ in the present study is uncertain because assessment of nonamnestic functions relied only on two executive measures despite the fact that nonamnestic forms of MCI also may include deficits in attention, language and visuospatial abilities [8]. Nordlund et al. [33] employed a more comprehensive neuropsychological assessment in a similar MCI population and found conversion to dementia, within a 2-year time span, only in multidomain amnestic and multidomain nonamnestic patients, and not in any form of single-domain MCI. Our findings might have been similar to the findings of Nordlund et al. [33] if we had included a more comprehensive nonamnestic assessment.

The incidence of dysexecutive MCI ranges from 3 to 15\% [18] in studies employing neuropsychological tests but with different inclusion criteria and diverse test batteries. In our sample, $11 \%$ of the neuropsychologically impaired patients fulfilled our criteria for dysexecutive MCI. Based on the ADNI study, Gibbons et al. [17] developed a composite score for executive functioning (constructed of 6 executive tests) and found this score to be a strong predictor of AD conversion after 3 years. In a much smaller longitudinal study by Whitwell et al. [16], 9 patients were characterized as attention/dysexecutive MCI cases. Of these, 6 progressed to dementia, 3 showed conversion to dementia with Lewy bodies, and 3 showed conversion to AD dementia. The prognoses for the patients with executive deficits in the ADNI study [17] as well as in the study by Whitwell et al. [16] differ from the prognoses for the 
patients in a similar cognitive category in our study, where 2 patients progressed to dementia and 7 reverted to normal neuropsychological function. Possibly the most important reason for this discrepancy is that, in the ADNI study [17], the mean age was 75 years, and in the study by Whitwell et al. [16], it was 78 years. In this age group, the prevalence of dementia is $5 \%$ [52], as opposed to a much lower risk of dementia in the present study group with a mean age of 63 years. In a study on younger MCI patients, Johnson et al. [13] identified 31 older adults with pure dysexecutive MCI. Twelve of the patients progressed clinically over a 2-year period, but only 2 to probable dementia, and none reverted to normal. Those who declined had a mean age of 68 years, while those who remained stable had a mean age of 60 years, close to the mean age of our group.

It is problematic to determine the risk of further decline in executive and other cognitive functions in relatively young people with subjective cognitive complaints or MCI. Dysexecutive symptoms may reflect different conditions with different neuropathologies and prognoses, or may represent normal variation in symptoms in healthy people [48]. Bassett and Folstein [52] found that $43 \%$ of those between 65 and 74 years report subjective memory problems, while the prevalence of dementia within this age range and also within older age ranges is minimal. To achieve a better understanding of the further course and etiology of relatively young people with mild executive and amnestic deficits, future studies should prolong the follow-up period and include CSF and genetic markers as well as MRI [13]. This study and many other MCI studies employed relatively short test batteries. This is practical, but the robustness of the cognitive findings may suffer as healthy participants may be categorized as cognitively impaired based on single and possibly incidental poor scores. To increase the robustness and specificity, as well as the ability to precisely categorize cognitive function in low-risk groups, future studies should also employ more comprehensive neuropsychological assessments [50] than the present study.

In conclusion, the present study found that executive or amnestic deficits in either their pure form or in combination occur frequently in a relatively young clinical population (mean age: 63 years) referred for cognitive complaints. During the study period, $10.5 \%$ of the patients with single-domain dysexecutive MCI converted to dementia, while $29.2 \%$ of the patients with amnestic MCI became demented. For patients with both executive and amnestic deficits, the risk of conversion to dementia was $59.6 \%$, clearly indicating that dysexecutive symptoms in combination with amnestic symptoms constitute a risk factor for dementia in young MCI patients.

\section{Disclosure Statement}

The authors report no conflicts of interest.

\section{References}

1 Weiner MW, Veitch DP, Aisen PS, et al: The Alzheimer's Disease Neuroimaging Initiative: a review of papers published since its inception. Alzheimers Dement 2013;9:e111-e194.

2 Hachinski V, Iadecola C, Petersen RC, et al: National Institute of Neurological Disorders and Stroke-Canadian Stroke Network vascular cognitive impairment harmonization standards. Stroke 2006;37:2220-2241.

-3 Petersen RC, Smith GE, Waring SC, Ivnik RJ, Tangalos EG, Kokmen E: Mild cognitive impairment: clinical characterization and outcome. Arch Neurol 1999;56:303-308.

4 Mitchell AJ, Shiri-Feshki M: Rate of progression of mild cognitive impairment to dementia: meta-analysis of 41 robust inception cohort studies. Acta Psychiatr Scand 2009;119:252-265.

5 Petersen RC: Mild cognitive impairment. N Engl J Med 2011;364:2227-2234. 
-6 Tabert MH, Manly JJ, Liu X, Pelton GH, Rosenblum S, Jacobs M, Zamora D, Goodkind M, Bell K, Stern Y, Devanand DP: Neuropsychological prediction of conversion to Alzheimer disease in patients with mild cognitive impairment. Arch Gen Psychiatry 2006;63:916-924.

-7 Dickerson BC, Sperling RA, Hyman BT, Albert MS, Blacker D: Clinical prediction of Alzheimer disease dementia across the spectrum of mild cognitive impairment. Arch Gen Psychiatry 2007;64:1443-1450.

8 Petersen RC, Roberts RO, Knopman DS, Boeve BF, Geda YE, Ivnik RJ, Jack CR Jr: Mild cognitive impairment: ten years later. Arch Neurol 2009;66:1447-1455.

9 Petersen RC, Aisen PS, Beckett LA, Donohue MC, Gamst AC, Harvey DJ, Weiner MW: Alzheimer's Disease Neuroimaging Initiative (ADNI): clinical characterization. Neurology 2010;74:201-209.

10 Chao LL, Pa J, Duarte A, Schuff N, Weiner MW, Kramer JH, Miller BL, Freeman KM, Johnson JK: Patterns of cerebral hypoperfusion in amnestic and dysexecutive MCI. Alzheimer Dis Assoc Disord 2009;23:245-252.

11 Grambaite R, Selnes P, Reinvang I, Aarsland D, Hessen E, Gjerstad L, Fladby T: Executive dysfunction in mild cognitive impairment is associated with changes in frontal and cingulate white matter tracts. J Alzheimers Dis 2011;27:453-462.

-12 Johnson JK, Vogt BA, Kim R, Cotman CW, Head E: Isolated executive impairment and associated frontal neuropathology. Dement Geriatr Cogn Disord 2004;17:360-367.

13 Johnson JK, Pa J, Boxer AL, Kramer H, Freeman K, Yaffe K: Baseline predictors of clinical progression among patients with dysexecutive mild cognitive impairment. Dement Geriatr Cogn Disord 2010;30:344-351.

14 Nordlund A, Rolstad S, Hellström P, Sjögren M, Hansen S, Wallin A: The Göteborg MCI study: mild cognitive impairment is a heterogeneous condition. J Neurol Neurosurg Psychiatry 2005;76:1485-1490.

15 Pa J, Boxer A, Chao LL, Gazzaley A, Freeman K, Kramer J, Miller BL, Weiner MW, Neuhaus J, Johnson JK: Clinicalneuroimaging characteristics of dysexecutive mild cognitive impairment. Ann Neurol 2009;65:414-423.

-16 Whitwell JL, Petersen RC, Negash S, Weigand SD, Kantarci K, Ivnik RJ, Knopman DS, Boeve BF, Smith GE, Jack CR Jr: Patterns of atrophy differ among specific subtypes of mild cognitive impairment. Arch Neurol 2007;64: 1130-1138.

17 Gibbons LE, Carle AC, Mackin RS, Harvey D, Mukherjee S, Insel P, Curtis SM, Mungas D, Crane PK: A composite score for executive functioning, validated in Alzheimer's Disease Neuroimaging Initiative (ADNI) participants with baseline mild cognitive impairment. Brain Imaging Behav 2012;6:517-527.

18 Jak AJ, Bangen KJ, Wierenga CE, Delano-Wood L, Corey-Bloom J, Bondi MW: Contributions of neuropsychology and neuroimaging to understanding clinical subtypes of mild cognitive impairment. Int Rev Neurobiol 2009; 84:81-103.

19 Farias ST, Mungas D, Reed BR, Harvey D, DeCarli C: Progression of mild cognitive impairment to dementia in clinic- vs community-based cohorts. Arch Neurol 2009;66:1151-1157.

20 Román G, Erkinjuntti T, Wallin A, Pantoni L, Chui H: Subcortical ischaemic vascular dementia. Lancet Neurol 2002;1:426-436.

-21 Grau-Olivares M, Arboix A: Mild cognitive impairment in stroke patients with ischemic cerebral small-vessel disease: a forerunner of vascular dementia? Expert Rev Neurother 2009;9:1201-1217.

-22 Rosen HJ, Lengenfelder J, Miller B: Frontotemporal dementia. Neurol Clin 2000;18:979-992.

23 Aarsland D, Brønnick K, Fladby T: Mild cognitive impairment in Parkinson's disease. Curr Neurol Neurosci Rep 2011;11:371-378.

24 Ferman TJ, Boeve BF, Smith GE, Silber MH, Kokmen E, Petersen RC, Ivnik RJ: REM sleep behavior disorder and dementia: cognitive differences when compared with AD. Neurology 1999;52:951-957.

25 Albert M, Blacker D, Moss MB, Tanzi R, McArdle JJ: Longitudinal change in cognitive performance among individuals with mild cognitive impairment. Neuropsychology 2007;21:158-169.

-26 Chen P, Ratcliff G, Belle SH, Cauley JA, DeKosky ST, Ganguli M: Cognitive tests that best discriminate between presymptomatic AD and those who remain nondemented. Neurology 2000;55:1847-1853.

27 Huntley JD, Howard RJ: Working memory in early Alzheimer's disease: a neuropsychological review. Int J Geriatr Psychiatry 2010;25:121-132.

28 Wilkins CH, Mathews J, Sheline YI: Late life depression with cognitive impairment: evaluation and treatment. Clin Interv Aging 2009;4:51-57.

29 Roman G, Erkinjuntti T, Wallin A, Pantoni L, Chui H: Subcortical ischemic vascular dementia. Lancet Neurol 2002;1:426-436.

-30 Huey ED, Manly JJ, Tang MX, Schupf N, Brickman AM, Manoochehri M, Mez J, DeCarli C, Devanand DP, Mayeux R: Course and etiology of dysexecutive MCI in a community sample. Alzheimers Dement 2013;9:632-639.

-31 Dubois B, Feldman HH, Jacova C, DeKosky ST, Barberger-Gateau P, Cummings J, Scheltens P: Research criteria for the diagnosis of Alzheimer's disease: revising the NINCDS-ADRDA criteria. Lancet Neurol 2007;6:734-746.

-32 Dubois B, Feldman HH, Jacova C, Cummings JL, DeKosky ST, Barberger-Gateau P, Scheltens P: Revising the definition of Alzheimer's disease: a new lexicon. Lancet Neurol 2010;9:1118-1127.

-33 Nordlund A, Rolstad S, Klang O, Edman A, Hansen S, Wallin A: Two year outcome of MCI subtypes and aetiologies in the Göteborg MCI study. J Neurol Neurosurg Psychiatry 2010;81:541-546.

34 Miyake A, Friedman NP, Emerson JM, Witzki AH, Howerter A, Wager TD: The unity and diversity of executive functions and their contributions to complex 'frontal lobe' tasks: a latent variable analysis. Cogn Psychol 2000; 41:49-100.

-35 Brandt J, Aretouli E, Neijstrom E, Samek J, Manning K, Albert MS, Bandeen-Roche K: Selectivity of executive function deficits in mild cognitive impairment. Neuropsychology 2009;23:607-618. 
-36 Johns EK, Phillips NA, Belleville S, Goupil D, Babins L, Kelner N, Ska B, Gilbert B, Massoud F, de Boysson C, Duncan HD, Chertkow $\mathrm{H}$ : The profile of executive functioning in amnestic mild cognitive impairment: disproportionate deficits in inhibitory control. J Int Neuropsychol Soc 2012;18:541-555.

-37 Grambaite R, Stenset V, Reinvang I, Walhovd KB, Fjell AM, Fladby T: White matter diffusivity predicts memory in patients with subjective and mild cognitive impairment and normal CSF total tau levels. J Int Neuropsychol Soc 2010;16:58-69.

38 Reisberg B, Ferris SH, de Leon MJ, Crook T: Global Deterioration Scale (GDS). Psychopharmacol Bull 1988;24: 661-663.

-39 Auer S, Reisberg B: The GDS/FAST staging system. Int Psychogeriatr 1997;9(suppl 1):167-171.

40 Rey A: L'examen clinique en psychologie. Paris, Presses Univeritaires de France, 1964.

41 Schmidt M: Rey Auditory and Verbal Learning Test: A Handbook. Los Angeles, Western Psychological Services, 1996.

42 Meyers JE, Meyers KR: Rey Complex Figure Test and Recognition Trial. Odessa, Psychological Assessment Resources, 1995.

43 Reitan RM, Wolfson D: The Halstead-Reitan Neuropsychological Test Battery. Tucson, Neuropsychology Press, 1985.

44 Benton AL, Hamsher K, Sivan AB: Multilingual Aphasia Examination. Iowa City, AJA Associates, 1989.

45 Heaton RK, Miller SW, Taylor MJ, Grant I: Revised Comprehensive Norms for an Expanded Halsted-Reitan Battery: Demographically Adjusted Neuropsychological Norms for African American and Caucasian Adults. Odessa, Psychological Assessment Resources, 2004.

-46 Petersen RC, Roberts RO, Knopman DS, Geda YE, Cha RH, Pankratz VS, Boeve BF, Tangalos EG, Ivnik RJ, Rocca WA: Prevalence of mild cognitive impairment is higher in men: the Mayo Clinic Study of Aging. Neurology 2010;75:889-897.

47 Harvey R: Young Onset Dementia: Epidemiology, Clinical Symptoms, Family Burden, Support and Outcome. London, Dementia Research Group, 1998.

48 Buckner RL: Memory and executive function in aging and AD: multiple factors that cause decline and reserve factors that compensate. Neuron 2004;44:195-208.

49 McCaffrey RJ, Duff K, Westerwelt HJ: Practitioners Guide to Evaluating Change with Neuropsychological Assessment Instruments. New York, Kluwer Academic/Plenum Publishers, 2000.

50 Lezak MD, Howieson DB, Bigler ED, Tranel D: Neuropsychological Assessment, ed 5. Oxford, Oxford UP, 2012.

-51 Bozoki A, Giordani B, Heidebrink JL, Berent S, Foster NL: Mild cognitive impairments predict dementia in nondemented elderly patients with memory loss. Arch Neurol 2001;58:411-416.

52 Bassett SS, Folstein MF: Memory complaint, memory performance, and psychiatric diagnosis: a community study. J Geriatr Psychiatry Neurol 1993;6:105-111. 